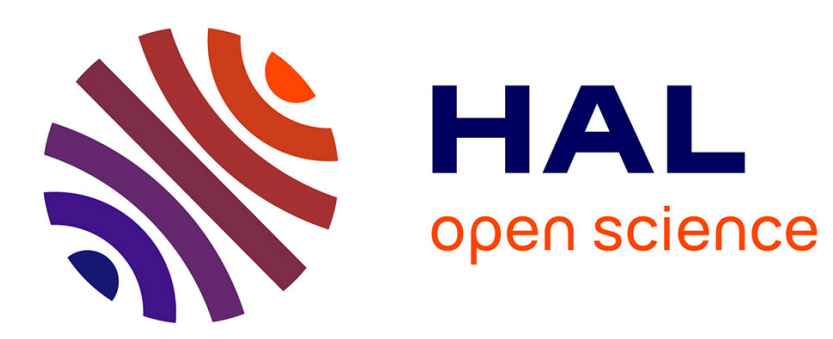

\title{
Structural reactivation in plate tectonics controlled by olivine crystal anisotropy
}

\author{
Andrea Tommasi, Mickael Knoll, Alain Vauchez, J. Signorelli, Catherine \\ Thoraval, Roland E. Logé
}

\section{- To cite this version:}

Andrea Tommasi, Mickael Knoll, Alain Vauchez, J. Signorelli, Catherine Thoraval, et al.. Structural reactivation in plate tectonics controlled by olivine crystal anisotropy. Nature Geoscience, 2009, 2 (6), pp.423-427. 10.1038/ngeo528 . hal-00413033

\section{HAL Id: hal-00413033 \\ https://hal.science/hal-00413033}

Submitted on 16 Jun 2021

HAL is a multi-disciplinary open access archive for the deposit and dissemination of scientific research documents, whether they are published or not. The documents may come from teaching and research institutions in France or abroad, or from public or private research centers.
L'archive ouverte pluridisciplinaire HAL, est destinée au dépôt et à la diffusion de documents scientifiques de niveau recherche, publiés ou non, émanant des établissements d'enseignement et de recherche français ou étrangers, des laboratoires publics ou privés. 


\title{
Structural reactivation in plate tectonics controlled by olivine crystal anisotropy
}

\author{
Andréa Tommasi ${ }^{1 \star}$, Mickael Knoll1,2, Alain Vauchez ${ }^{1}$, Javier W. Signorelli ${ }^{3}$, Catherine Thoraval ${ }^{1}$ \\ and Roland Logéz
}

\begin{abstract}
Reactivation of structures inherited from previous collisional or rifting events, especially lithospheric-scale faults, is a major feature of plate tectonics. Its expression ranges from continental break-up along ancient collisional belts ${ }^{1,2}$ to linear arrays of intraplate magmatism and seismicity ${ }^{3,4}$. Here we use multiscale numerical models to show that this reactivation can result from an anisotropic mechanical behaviour of the lithospheric mantle due to an inherited preferred orientation of olivine crystals. We explicitly consider an evolving anisotropic viscosity controlled by the orientation of olivine crystals in the mantle. We find that strain is localized in domains where shear stresses on the inherited mantle fabric are high, and that this leads to shearing parallel to the inherited fabric. During rifting, structural reactivation induced by anisotropy results in oblique extension, followed by either normal extension or failure. Our results suggest that anisotropic viscosity in the lithospheric mantle controls the location and orientation of intraplate deformation zones that may evolve into new plate boundaries, and causes long-lived lithospheric-scale wrench faults, contributing to the toroidal component of plate motions on Earth.
\end{abstract}

Texture-induced viscoplastic anisotropy is an intrinsic feature of crystalline materials deforming by dislocation creep. It entails a directional dependence of the mechanical strength on the crystal orientation. This behaviour, expressed macroscopically as an anisotropic viscosity, results from both the anisotropy of the elastic tensor and the discrete nature of dislocation glide along densely packed lattice directions on selected crystallographic planes. It depends on the crystal symmetry and, at the aggregate scale, on the orientation of the constitutive crystals or texture. As they have fewer slip systems, low-symmetry minerals are more anisotropic than cubic metals. Orthorhombic olivine is highly anisotropic: it has only three independent slip systems. At high temperature, an olivine crystal deforms up to 100 times faster if it activates the 'easy' (010)[100] system rather than the 'hard' (010)[001] system ${ }^{5,6}$. This leads to development of strong crystal preferred orientations (texture), which are ubiquitous in naturally and experimentally deformed mantle rocks ${ }^{7}$. Moreover, seismic anisotropy provides evidence for coherent orientation of olivine crystals at the scale of hundreds of kilometres in the upper mantle ${ }^{8}$. Yet, in contrast to the large number of studies on the role of texture-induced plastic anisotropy on the deformation of cubic and hexagonal metallic alloys since the early work of ref. 9, plastic anisotropy has been largely ignored in earth sciences.

Models considering a transversely isotropic viscosity showed nevertheless that anisotropy changes the convection length scales, leads to strain localization, and modifies the initiation times of convective instabilities in the mantle $\mathrm{e}^{10-12}$. Mechanical anisotropy due to olivine crystal preferred orientations in the mantle was also proposed to explain the reactivation of ancient collisional structures during continental rifting ${ }^{2}$. Models of the deformation of a homogeneous, anisotropic mantle subjected to an axisymmetric tension show a strong directional softening, leading to strain localization and development of shearing when extensional stresses are oblique to the pre-existing mantle fabric ${ }^{13}$. These models avoided simplifications to the viscosity tensor by using a viscoplastic self-consistent (VPSC) formalism ${ }^{14}$ to relate the crystal and largescale plastic anisotropies, but they did not allow for the evolution of olivine textures and, hence, of the anisotropy in response to the new solicitation.

To fully account for an evolving anisotropic viscosity as a function of the orientation of olivine crystals in the mantle, we coupled the VPSC formalism to FORGE2005, a commercialized three-dimensional finite-element mechanical (FEM) code. The coupling is performed through a set of 1,000 olivine crystals associated with each finite element. After each FEM time increment, the local velocity gradient tensor is used as a boundary condition for the VPSC simulation, leading to evolution of the polycrystal texture and viscosity. The updated polycrystal viscosity tensor is then used to calculate the stress field in the next FEM time step (see Supplementary Fig. S1).

To analyse the deformation of a plate containing a pre-existing lithospheric-scale transcurrent shear zone or transpressional belt, we run a series of models using a plate with aspect ratio $1.2 \times 1 \times 0.1$ (Fig. 1) subjected to a constant divergent velocity or tensional stress on its left lateral boundary. Free-slip conditions (normal velocities and shear stresses are null) are imposed on the opposite boundary. Boundaries normal to the $y$ and $z$ axes are free (normal and tangential stresses are null). We use a multidomain meshing tool to define a planar zone, 0.1 or 0.2 units wide, cross-cutting the entire plate, at $45^{\circ}$ (Fig. 1) or normal to the $x$ axis (see Supplementary Fig. S2). This zone has an initial 'wrench-fault-type' olivine texture ${ }^{15}$; maximum concentrations of [100] and [010] axes are horizontal, respectively parallel and normal to the shear-zone trend (Fig. 1). This texture is coherent with shear-wave splitting data in large-scale strike-slip faults and transform boundaries, where fast $S$ waves are polarized parallel to the trend of the fault up to $50 \mathrm{~km}$ away from the surface expression of the fault ${ }^{16}$, and in collisional belts ${ }^{8,15,17}$. For numerical stability reasons, the initial texture is however significantly weaker $\left(J_{\text {index }}=3\right.$, Fig. 1 , where $J_{\text {index }}$ is a measure of the texture intensity, expressed as the volume-averaged integral of the squared orientation densities) than

\footnotetext{
${ }^{1}$ Geosciences Montpellier, CNRS \& Université de Montpellier 2, PI. E Bataillon, 34095 Montpellier cedex 5, France, ${ }^{2}$ MINES ParisTech, CEMEF-Center for Materials Forming, UMR CNRS 7635, BP207, 06904 Sophia Antipolis Cedex, France, ${ }^{3}$ Instituto de Fisica de Rosario, CONICET, Universidad Nacional de Rosario, 2000 Rosario, Argentina. *e-mail: andrea.tommasi@gm.univ-montp2.fr.
} 


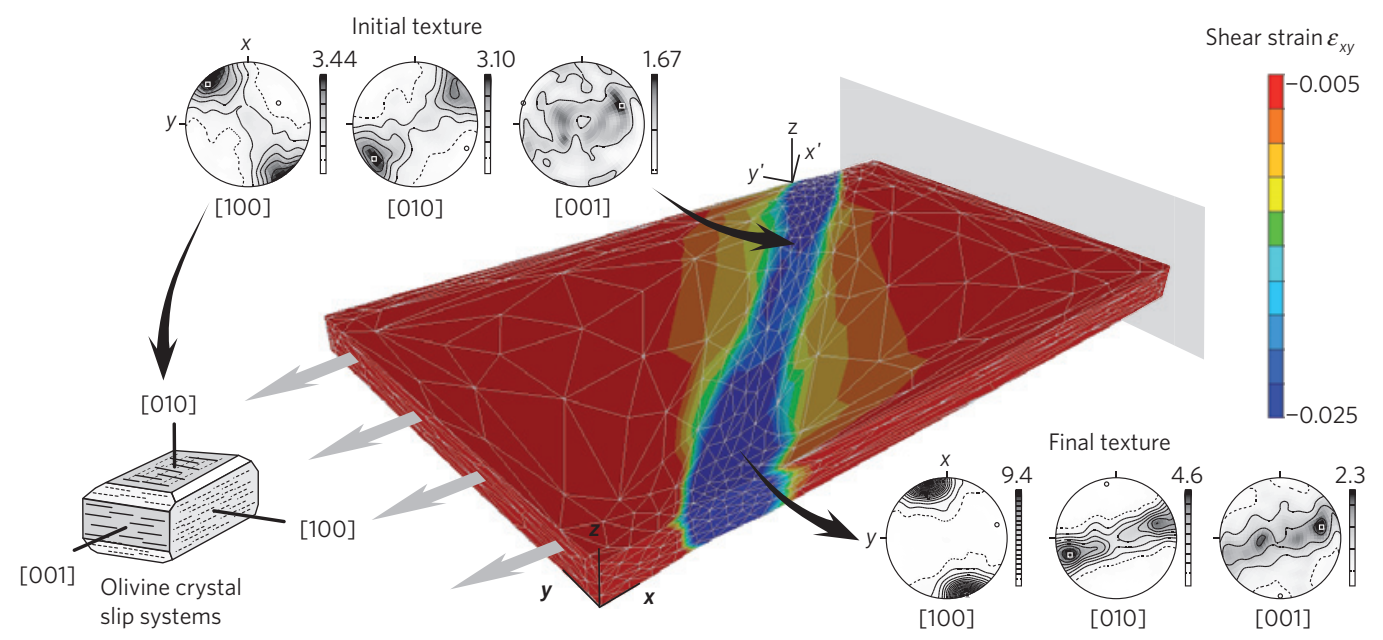

Figure 1 | Geometry, boundary conditions and shear-strain $\varepsilon_{x y}$ distribution in a model with an 'inherited shear zone' at $45^{\circ}$ of the imposed extension direction after a total stretching of $\mathbf{4 0} \%$. Texture-induced anisotropy results in strain localization and higher shear strain in the 'inherited shear zone'. Evolution of the olivine texture within the 'inherited shear zone' may be evaluated by comparing the initial (top left) and final (bottom right) textures. Bottom left: slip systems in the olivine crystal, 'easy' (010)[100] and (001)[100] systems are outlined by dark-grey shading and thicker lines parallel to the slip direction.

a

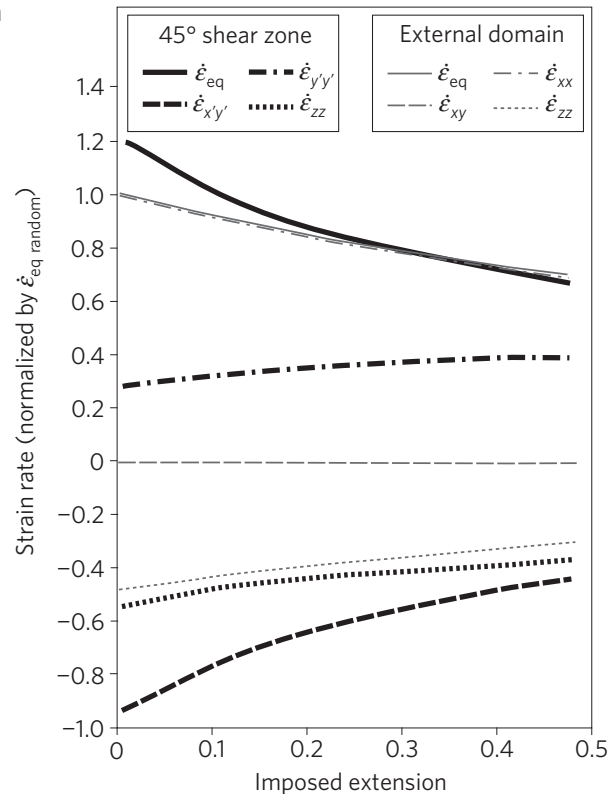

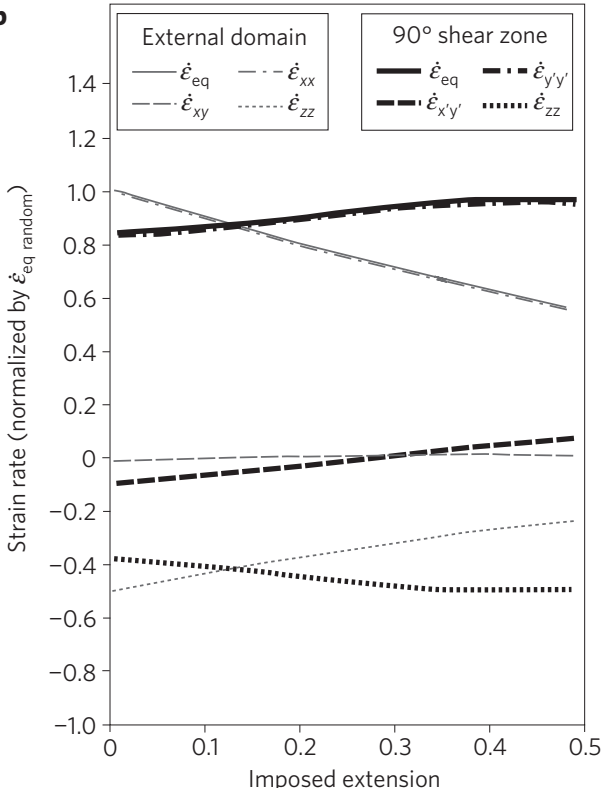

Figure 2 | Evolution of strain rates in the inherited texture zone (thick lines) and in the surroundings (thin lines) as a function of the imposed extension. $\mathbf{a}$, Inherited 'shear zone' at $45^{\circ}$ to the imposed extension. $\mathbf{b}$ Inherited 'shear zone' normal to the imposed extension. Analysis of the Von Mises equivalent strain rate $\dot{\varepsilon}_{\text {eq }}=\sqrt{2 / 3 \dot{\varepsilon}_{i j} \dot{\varepsilon}_{i j}}$ highlights the evolution of the strain distribution in response to the reorientation of the olivine crystal preferred orientations in both domains. The latter also results in changes in the deformation regime, outlined by the evolution of the rates of shearing parallel to the inherited shear zone $\left(\dot{\varepsilon}_{x^{\prime} y^{\prime}}\right)$, extension normal to it $\left(\dot{\varepsilon}_{y^{\prime} y^{\prime}}\right)$ and vertical thinning $\left(\dot{\varepsilon}_{z z}\right)$.

those from naturally deformed peridotites ${ }^{7}\left(2<J_{\text {index }}<26\right.$, peak at 8 ). Olivine texture in the surrounding medium is initially random.

All models show an anisotropic behaviour, characterized by dependence of strength on the direction of solicitation relative to the texture orientation. Strain localization arises owing to lateral variations in texture orientation and intensity. Equivalent strain rates $\left(\dot{\varepsilon}_{\text {eq }}\right)$ are higher when the texture orientation relative to the imposed extension results in high resolved shear stresses on the 'easy' (010)[100] and (001) [100] slip systems in most crystals within a domain. Strain is therefore localized in the 'inherited texture domain' at $45^{\circ}$ to the imposed extension (Figs 1 and 2a), whereas a similar domain normal to the extension has a higher initial strength, deforming more slowly than the surrounding medium (Fig. 2b).
Decomposition of the horizontal flow field into its poloidal (divergence) and toroidal (vorticity) components highlights that viscoplastic anisotropy produces lateral variations in the poloidal flow field (strain localization) and toroidal flow (strike-slip deformation) in the 'inherited texture domain' (Fig. 3). The latter deforms therefore by transtension (Fig. 2): shearing parallel to its trend $\left(\dot{\varepsilon}_{x^{\prime} y^{\prime}}\right)$ accompanies stretching normal to it $\left(\dot{\varepsilon}_{y^{\prime} y^{\prime}}\right)$ and vertical thinning $\left(\dot{\varepsilon}_{z z}\right)$. The intensity of the toroidal component depends to the first order on the orientation of the texture relative to the stress field, but also on the texture intensity. It is higher in 'inherited texture domains' oblique to the extension direction and for stronger initial textures (see Supplementary Fig. S3). Analysis of the results of the model with an 'inherited texture domain' normal to the 
a

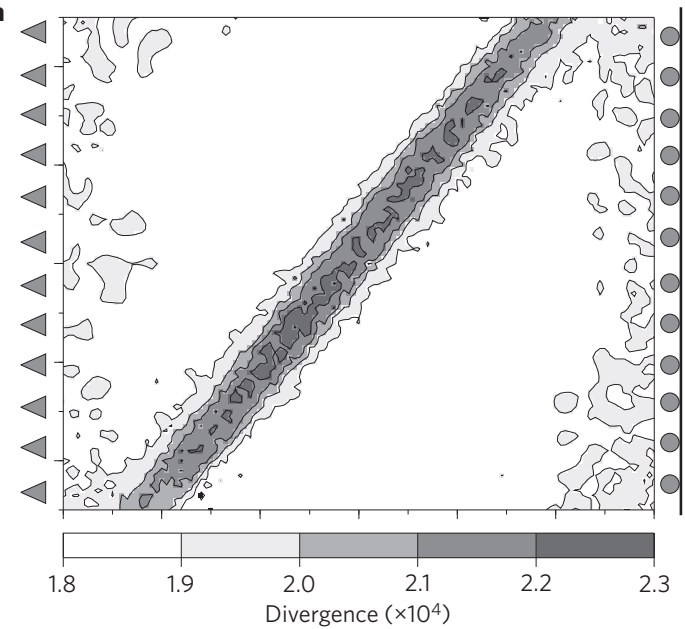

c

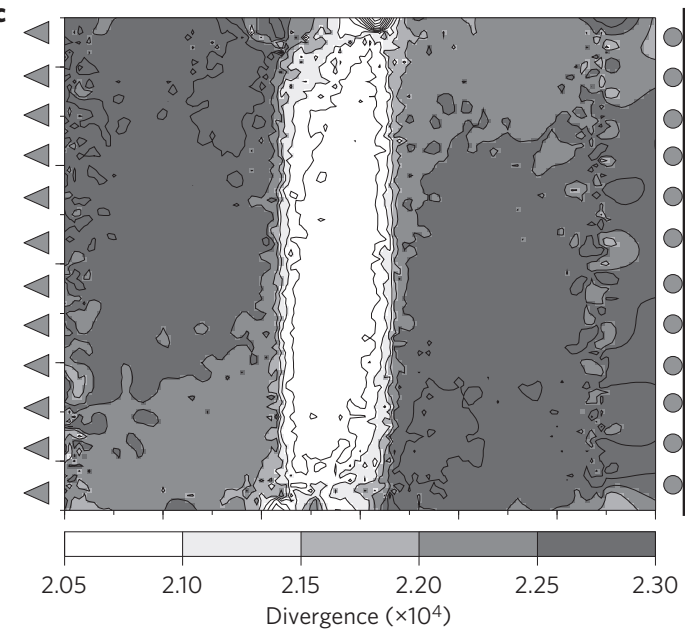

b

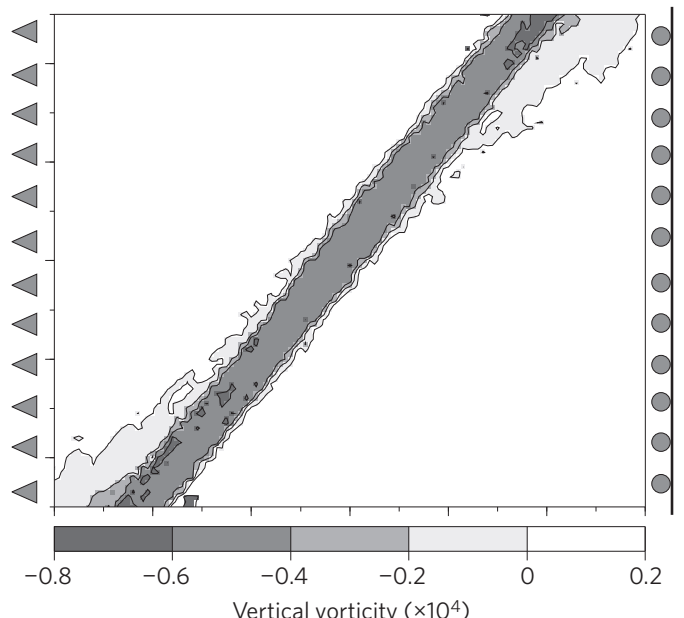

d

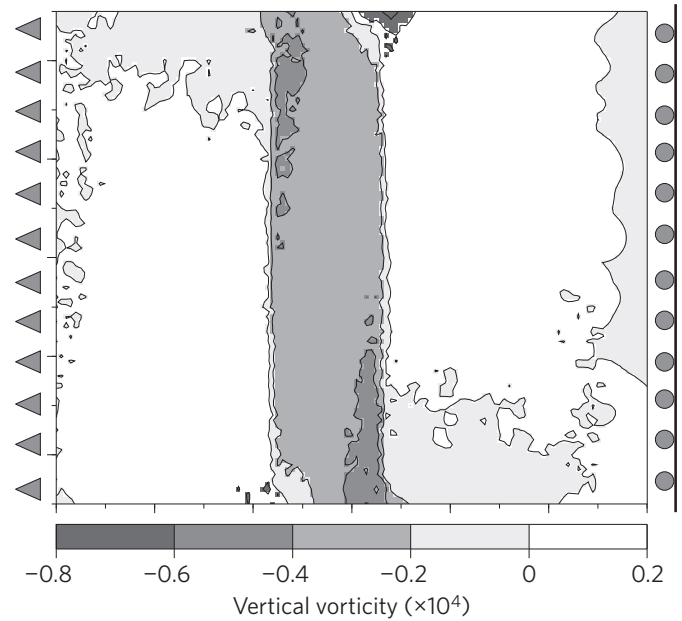

Figure $\mathbf{3}$ | Poloidal-toroidal partitioning in the models. Maps of the divergence $(\mathbf{a}, \mathbf{c})$ and vorticity $(\mathbf{b}, \mathbf{d})$ of the horizontal flow field. Higher divergence values indicate strain localization in the 'inherited texture domain' or in the surroundings, when the former is at $45^{\circ}$ (a) or normal (b) to the imposed extension. Larger divergence variations denote stronger localization in the $45^{\circ}$ model. Toroidal flow, indicated by a non-null vorticity, is restricted to the 'inherited texture domain'. Divergence and vorticity variations are directly related to changes in the olivine texture, being roughly homogeneous within each domain.

extension shows however that even a slight departure from perfect symmetry of the texture (see Supplementary Fig. S2) produces toroidal flow (Fig. 3) and hence horizontal shearing within the inherited texture domain (Fig. 2b).

Evolution of olivine textures with increasing strain modifies the strain distribution. Progressive rotation of olivine [100] and [010] axes towards the maximum finite extension and shortening directions, respectively (Fig. 1), results in geometrical hardening in both the 'inherited texture domain' and the surroundings (Fig. 2), because shear stresses on the 'weak' (010)[100] and (001)[100] slip systems decrease. Faster texture evolution in the inherited texture domain' in the $45^{\circ}$ model (the surroundings in the $90^{\circ}$ one) results in rehomogenization of the strain distribution (inversion of the strength contrast) at high strain. Texture evolution and hence the mechanical behaviour depend on the strain regime: extension or compression normal to the dominant orientation of the easy slip systems produce hardening, whereas shearing parallel to it produces softening (see Supplementary Fig. S4). Reorientation of the olivine texture also changes the deformation regime in the 'inherited texture domain' (Fig. 2). In the $45^{\circ}$ model, the shear rate parallel to the trend of the 'inherited texture domain' $\left(\dot{\varepsilon}_{x^{\prime} y^{\prime}}\right)$ decreases and the extension rate normal to it $\left(\dot{\varepsilon}_{y^{\prime} y^{\prime}}\right)$ increases; the deformation evolves from transtensional to extensional (Fig. 2a).

Although extremely simple (they do not consider thermal effects and test only end-member geometries), these models show that viscoplastic anisotropy due to preferred orientation of olivine crystals triggers the reactivation of pre-existing lithospheric-scale faults or transpressional belts if their orientation enables higher shear stresses on the olivine crystals' easy slip systems within them than in the surroundings. Strain localization in the present models is limited: the strain-rate contrast between the domain with inherited texture and the initially isotropic surroundings is less than 2 (Fig. 2). This contrast is enhanced if the inherited texture is stronger, like those usually observed in naturally deformed peridotites (see Supplementary Fig. S3), or if the surroundings have a non-random initial texture in a 'hard' orientation relatively to the solicitation, but texture-induced mechanical anisotropy remains less effective in producing strain localization than changes in deformation mechanism due to grain refinement ${ }^{18}$, viscous heating ${ }^{19}$ or damage processes ${ }^{20}$. However, the latter are observed neither in hightemperature, high-pressure experiments nor in nature and the former processes require an already localized forcing, that is, a pre-existing localization of the deformation ${ }^{21}$, usually produced in geodynamical models by the introduction of ad hoc temperature anomalies or 'weak domains'. Viscoplastic anisotropy due to coherent orientation of olivine crystals, in contrast, is an intrinsic feature of lithospheric plates as shown by shear-wave splitting ${ }^{8}$.

We propose that lateral variations in mechanical behaviour due to changes in the inherited olivine crystal preferred orientations within a plate represent a major 'seed' process for strain localization 
creating the conditions for the activation of additional efficient localizing processes. In contrast to lateral variations of the geotherm, crustal thickness, or damage zones associated with brittle faults that may also seed localization, its lifetime is not limited by heat diffusion, isostatic re-equilibration, or erosion. Coherence between crustal structures of all ages and seismic anisotropy data suggest that texture-induced anisotropy may be preserved for more than 1 Gyr. It may therefore explain the reactivation of lithosphericscale strike-slip or transpressional zones hundreds of Myr old, as observed in the South Atlantic and East African rifting ${ }^{2,13}$.

Deformation controlled by texture-induced viscoplastic anisotropy associated with lithospheric-scale wrench faults and transpressional belts has a characteristic signature: development of shearing parallel to the trend of the reactivated structures. Rift initiation controlled by anisotropy-induced reactivation of shear zones results in transtension followed by normal extension. This evolution is observed in the East African rift ${ }^{22}$, the North Atlantic margins ${ }^{23}$, the Rhine graben ${ }^{24}$ and the eastern Gondwana break-up ${ }^{25}$. It supports reactivation of neoproterozoic belts during the eastern Gondwana and East African rifting, of Caledonian structures in the North Atlantic opening and of Variscan wrench faults in the Rhine graben. It also suggests that the role of mechanical anisotropy decreases in mature rift systems, in agreement with the present models' prediction that evolution of the olivine texture leads to progressive geometric hardening and, hence, to rift failure, if thermo-mechanical or magmatic processes leading to further softening are not activated in the thinned lithospheric domain.

As it favours shearing parallel to the pre-existing mantle structure, viscoplastic anisotropy may also explain the reactivation of large-scale wrench faults during successive collisional and extensional episodes, such as the Newfoundland-Azores-Gibraltar fault zone, which acted as a dextral strike-slip boundary during the Hercynian orogeny ${ }^{26}$ and was reactivated as a major transform accommodating the differential motion between Africa and Europe during the Central Atlantic opening. The large proportion of strike-slip focal mechanisms in intraplate seismic arrays, such as the New Madrid seismic zone in the southeastern US (ref. 27) or the present seismic activity along the Hercynian South Armorican shear zone in France ${ }^{28}$, also suggests that the reactivation of the NE-SW- and NW-SE-trending mantle fabric imaged by shear-wave splitting in the southeastern US (ref. 29) and Brittany ${ }^{30}$ probably has a significant role in this intraplate deformation process.

A major consequence of viscoplastic anisotropy is that the stress and strain tensor eigenvectors are parallel only when the olivine texture is perfectly symmetric relatively to the stress field. Even a slight departure from symmetry produces shearing parallel to the average orientation of the dominant olivine slip system. Reactivation of a strike-slip or transpressional mantle texture therefore produces toroidal flow. The toroidal-poloidal partitioning depends on the orientation of the texture relative to the solicitation. In the present models (Fig. 3), the ratio between the vorticity and the divergence of the horizontal velocity field ranges from 0.2 to 0.3 ( 0.7 for a stronger initial olivine texture, Supplementary Fig. S3). These values encompass the average toroidal-poloidal partitioning ratio in the Earth (0.3-0.4; ref. 31), suggesting that texture-induced viscoplastic anisotropy in the mantle may significantly contribute to the Earth mantle toroidal flow and to belt-parallel transport recorded by both strain partitioning in the crust and fast-shear-wave polarization in past and present convergent boundaries ${ }^{17}$.

\section{Methods}

A VPSC formalism ${ }^{14}$ is used to relate the crystal and polycrystal mechanical behaviours and to predict the texture evolution. Interaction between the crystal and the polycrystal is calculated using the Eshelby formalism; each crystal is considered as an inclusion embedded in a homogeneous equivalent medium that behaves as the average of all crystals. At the crystal scale, deformation is accommodated by dislocation glide on discrete slip systems, whose relative strength-or critical resolved shear stress-depends on the temperature, pressure and chemical environment. In the present simulations, critical resolved shear stresses $\left(\tau_{0}\right)$ and stress exponents $(n)$ derived from high-temperature experiments on dry olivine crystals ${ }^{5,6}$ were used: $\tau_{0}^{[100](010)}=\tau_{0}^{[100](001)}=1 / 2 \tau_{0}^{[001](010)}=1 / 3 \tau_{0}^{[001](100)}$ and $n=3$ for all systems. Two additional slip systems, which are not observed in olivine, $\{\overline{1} 11\}\langle 110\rangle$ and $\{111\}\langle 110\rangle$, with $\tau_{0}=50 * \tau_{0}^{[100](010)}$, are added to ensure convergence. They never accommodate more than $1 \%$ of the total strain.

Three-dimensional FEM modelling. FORGE2005 is a commercialized FEM software using an updated Lagrangian scheme. The finite-element formulation is based on a mixed velocity-pressure formulation with an enhanced $(P 1+/ P 1)$ four-node tetrahedral element. Thermal effects are not considered. The mesh used in the present simulations is composed of about 12,000 linear tetrahedral elements. To define regions showing different initial textures, we use a multidomain mesh tool based on a level-set function ${ }^{32}$. Heterogeneous mesh refinement allows a fine description of the interfaces with a minimum number of mesh elements. Resolution tests using about 9,000,12,000, and 25,000 linear elements corroborate that strain localization does not depend on the mesh; the strain rate is roughly constant within each domain (small variations are observed close to the free boundaries) and the ratio between the average equivalent strain rates in the inherited texture domain and in the surroundings are similar. Low-resolution tests with about 4,500 elements show in contrast significantly weaker strain localization. To enable comparison between models, strain rates are normalized by the initial Von Mises equivalent strain rate, $\dot{\varepsilon}_{\text {eq }}=\sqrt{2 / 3 \dot{\varepsilon}_{i j} \dot{\varepsilon}_{i j}}$, of the surrounding medium, which has an initially random texture and hence an isotropic behaviour.

Received 4 February 2009; accepted 24 April 2009; published online 24 May 2009

\section{References}

1. Wilson, J. T. Did the Atlantic close and then re-open? Nature 211 676-681 (1966).

2. Vauchez, A., Barruol, G. \& Tommasi, A. Why do continents break up parallel to ancient orogenic belts? Terra Nova 9, 62-66 (1997).

3. Sykes, L. R. Intraplate seismicity, reactivation of preexisting zones of weakness, alkaline magmatism, and other tectonism postdating continental fragmentation. Rev. Geophys. Space Phys. 13, 621-688 (1978).

4. Zoback, M. D. et al. Recurrent intraplate tectonism in the New Madrid Seismic Zone. Science 209, 971-976 (1980).

5. Durham, W. B. \& Goetze, G. Plastic flow of oriented single crystals of olivine. 1. Mechanical data. J. Geophys. Res. 82, 5737-5753 (1977).

6. Bai, Q., Mackwell, S. J. \& Kohlstedt, D. L. High-temperature creep of olivine single crystals. 1. Mechanical results for buffered samples. J. Geophys. Res. 96, 2441-2463 (1991).

7. Tommasi, A., Mainprice, D., Canova, G. \& Chastel, Y. Viscoplastic self-consistent and equilibrium-based modelling of olivine lattice preferred orientations. Implications for upper mantle seismic anisotropy. J. Geophys. Res. 105, 7893-7908 (2000).

8. Wuestefeld, A., Barruol, G. \& Bokelmann, G. Shear-wave splitting database; available at <http://www.gm.univ-montp2.fr/splitting/ >.

9. Hill, R. A theory of yielding and plastic flow of anisotropic metals. Proc. $R$ Soc. Lond. A 193, 281-297 (1948).

10. Christensen, U. Some geodynamical effects of anisotropic viscosity. Geophys. J. R. Astron. Soc. 91, 711-736 (1987).

11. Honda, S. Strong anisotropic flow in a finely layered asthenosphere. Geophys. Res. Lett. 128, 1454-1457 (1986).

12. Lev, E. \& Hager, B. H. Rayleigh-Taylor instabilities with anisotropic lithospheric viscosity. Geophys. J. Int. 173, 806-814 (2008).

13. Tommasi, A. \& Vauchez, A. Continental rifting parallel to ancient collisional belts: An effect of the mechanical anisotropy of the lithospheric mantle. Earth Planet. Sci. Lett. 185, 199-210 (2001).

14. Lebensohn, R. A. \& Tomé, C. N. A self-consistent anisotropic approach for the simulation of plastic deformation and texture development of polycrystals: Application to zirconion alloys. Acta Metall. Mater. 41, 2611-2624 (1993).

15. Tommasi, A., Tikoff, B. \& Vauchez, A. Upper mantle tectonics: Three-dimensional deformation, olivine crystallographic fabrics and seismic properties. Earth Planet. Sci. Lett. 168, 173-186 (1999).

16. Vauchez, A. \& Tommasi, A. in Intraplate Strike-Slip Deformation Belts (eds Storti, F., Holdsworth, R. E. \& Salvini, F.) 15-24 (Special Publication, Vol. 210, Geological Society of London, 2003).

17. Vauchez, A. \& Nicolas, A. Mountain building: Strike-parallel displacements and mantle anisotropy. Tectonophysics 185, 183-201 (1991).

18. Braun, J. et al. A simple parameterization of strain localization in the ductile regime. J. Geophys. Res. 104, 25167-25181 (1999).

19. Regenauer-Lieb, K. \& Yuen, D. A. Positive feedback of interacting ductile faults from coupling of equation of state, rheology and thermal-mechanics. Phys. Earth Planet. Int. 142, 113-135 (2004). 
20. Bercovici, D. \& Ricard, Y. Tectonic plate generation and two-phase damage: Void growth versus grain size reduction. J. Geophys. Res. 110, B03401 (2005).

21. Montési, L. G. J. \& Zuber, M. T. A unified description of localization for application to large-scale tectonics. J. Geophys. Res. 107, 2045 (2002)

22. Bosworth, W. \& Strecker, M. R. Stress field changes in the Afro-Arabian rift system during the Miocene to Recent period. Tectonophysics 278, 47-62 (1997).

23. Geoffroy, L., Bergerat, F. \& Angelier, J. Tectonic evolution of the Greenland-Scotland Ridge during the Paleogene; new constraints. Geology 22, 653-656 (1994).

24. Schumacher, M. E. Upper Rhine Graben: Role of preexisting structures during rift evolution. Tectonics 21, 1-17 (2002).

25. Powell, C. M. A., Roots, S. R. \& Veewers, J. J. Pre-breakup continental extension in East Gondwanaland and the early opening of the eastern Indian Ocean. Tectonophysics 155, 261-283 (1988).

26. Keppie, J. D. in Terranes in the Circum-Atlantic Paleozoic Orogens (ed. Dallmeyer, R. D.) 159-192 (Special Paper, Vol. 230, Geological Society of America, 1989).

27. Herrmann, R. B. \& Canas, J.-A. Focal mechanism studies in the New Madrid seismic zone. Bull. Seismol. Soc. Am. 68, 1095-1102 (1978).

28. Mazabraud, Y., Bethoux, N., Guilbert, J. \& Bellier, O. Evidence for short-scale stress field variations within intraplate central-western France. Geophys. J. Int. 160, 161-178 (2005)

29. Barruol, G., Silver, P. G. \& Vauchez, A. Seismic anisotropy in the eastern United States: Deep structure of a complex continental plate. J. Geophys. Res. 102, 8329-8348 (1997).

30. Judenherc, S., Granet, M., Brun, J.-P. \& Poupinet, G. The Hercynian collision in the Armorican Massif; evidence of different lithospheric domains inferred from seismic tomography and anisotropy. Bull. Soc. Geol. Fr. 174, 45-57 (2003).
31. Lithgow-Bertelloni, C., Richards, M., Ricard, Y., O’Connell, R. J. \& Engebretson, D. C. Toroidal-poloidal partitioning of plate motions since 120 Ma. Geophys. Res. Lett. 20, 375-378 (1993).

32. Bernacki, M., Chastel, Y., Coupez, T. \& Logé, R. Level set framework for the numerical modelling of primary recrystallization in polycrystalline materials. Scripta Mater. 58, 1129-1132 (2008).

\section{Acknowledgements}

This study was partially funded by the programme Action Marges of the Institut National des Sciences de l'Univers, Centre National de la Recherche Scientifique (INSU-CNRS), France. Collaboration with J.W.S. was supported by a CNRS-CONICET cooperation program. M.K. benefited from a PhD scholarship from the Ministère de la Recherche et de l'Enseignement Supérieur, France.

\section{Author contributions}

This work is the outcome of a study on the effect of olivine fabrics on the mechanical behaviour of the continental lithosphere started by A.V. and A.T. M.K. ran all simulations as part of his Ph.D. under the supervision of A.T. and R.L. J.W.S. performed the coupling between the VPSC and the FEM codes. C.T. assisted in the analysis of the modelled flow fields.

\section{Additional information}

Supplementary information accompanies this paper on www.nature.com/naturegeoscience. Reprints and permissions information is available online at http://npg.nature.com/ reprintsandpermissions. Correspondence and requests for materials should be addressed to A.T. 